\title{
Dipole Moments of Isotactic and Syndiotactic Poly(methyl methacrylate) and Their Temperature Dependence
}

\author{
Mikiko Shima, Mari Sato, Miharu Atsumi, \\ and Koichi HATADA* \\ Faculty of Liberal Arts and Sciences, Tokyo Woman's Christian University, \\ Suginami, Tokyo 167, Japan \\ * Faculty of Engineering of Science, Osaka University, \\ Toyonaka, Osaka 560, Japan
}

(Received September 9, 1993)

\begin{abstract}
Dipole moments of isotactic (iso) and syndiotactic (syn) poly(methyl methacrylate) (PMMA) samples were determined from measurements of dielectric constants and densities in dilute benzene solution over the temperature range from 25 to $55^{\circ} \mathrm{C}$. The dipole moments and their temperature coefficients showed marked tacticity dependence. It was found that the iso-PMMA gives greater values of the dipole moment than those for the syn-PMMA; e.g., 1.457 D ( $=\mu$, dipole moment per constitutional repeating unit) was obtained for the iso-PMMA, and 1.323 D for the syn-PMMA at $25^{\circ} \mathrm{C}$. With increasing temperature, the dipole moment of the iso-PMMA showed little change and remained nearly constant, while that of the syn-PMMA increased continuously, giving $1.399 \mathrm{D}$ at $55^{\circ} \mathrm{C}$. Experimental dipole moment ratios, $D_{\mathrm{r}}=$ $\left\langle m^{2}\right\rangle / n \mu_{0}^{2}=\left\langle\mu^{2}\right\rangle / \mu_{0}^{2},\left(m\right.$, dipole moment of polymer; $\mu_{0}$, dipole moment of the constitutional unit; $n$, the number of units;) were compared with the calculated ones, which leads to the conclusion that rotational conditions regarding the orientation of polar side groups in the PMMA chains are highly restricted.
\end{abstract}

KEY WORDS Poly(methyl methacrylate) / Tacticity / Dipole Moment Ratio / Temperature Coefficient / Conformation / Dilute Solution /

The mean square dipole moment $\left\langle m^{2}\right\rangle$ of polymers having polar groups in their constitutional units is a conformation-dependent property like as the unperturbed chain dimension $\left\langle r_{0}^{2}\right\rangle$. Experimental values of the dipole moment can be determined from dielectric constant measurements in solution while theoretical values of $\left\langle m^{2}\right\rangle$ may be calculated using the "rotational isomeric state" model from structural information and conformational energies. Thus, study of dipole moments should provide information regarding the spatial configuration of polymers having polar groups.

Dipole moments of poly(methyl methacrylate) (PMMA) have long been studied, and the results of experimental studies have been reported numerously. ${ }^{1-6}$ The value of $\left\langle m^{2}\right\rangle$ of isotactic (iso) PMMA may be different from that of syndiotactic (syn) PMMA, because the conformation of chain molecules should depend on stereochemical constitution of the chain. The dependence of $\left\langle m^{2}\right\rangle$ of PMMA on tacticity was studied experimentally, in the 1960s, by Pohl et al. ${ }^{4}$ Salovey, ${ }^{5}$ and Roig et $a l .{ }^{6}$. More recently, theoretical studies on conformational statistics of iso- and synPMMA chains were reported by Flory, Sundararajan, and coworker. ${ }^{8-10}$ The dipole moments of iso- and syn-PMMAs were calculated by Birshtein et al. ${ }^{11}$. However, the reliability of experimental values of the dipole moments previously reported is apparently not 
high enough to be compared with the calculated ones, because of uncertainty of the tacticity of the samples and the accuracy of dielectric measurements was not satisfactorily high in former times. The progress in the anionic, stereospecific polymerization of methacrylates in the last decades has made it possible to prepare PMMA samples with well-defined structures and high tacticities. ${ }^{2-16}$ As previously described elsewhere ${ }^{7}$ the accuracy of dielectric measurements has also been improved. The dipole moment of polymers such as PMMA could be determined with an accuracy of $\pm 2 \times 10^{-3} \mathrm{D}$ ( $\mathrm{D}$, debye;), which is one factor higher than the former one.

The purpose of this study was to determine reliable experimental values of the dipole moments of PMMA with well defined tacticities, and get a knowledge of the conformations of iso- and syn-PMMA chains by comparing the experimental results with those calculated.

\section{EXPERIMENTAL}

\section{Polymer Samples}

Iso-PMMA was prepared in toluene at $-60^{\circ} \mathrm{C}$, using $t-\mathrm{C}_{4} \mathrm{H}_{9} \mathrm{MgBr}$ as an initiator, ${ }^{13,14}$ and syn-PMMA in toluene at $-78^{\circ} \mathrm{C}$, using $t-\mathrm{C}_{4} \mathrm{H}_{9} \mathrm{Li}-\left(\mathrm{C}_{2} \mathrm{H}_{5}\right)_{3} \mathrm{Al} .{ }^{15,16}$ The tacticities of the two PMMA samples determined by ${ }^{1} \mathrm{H}$ NMR measurements are $m m=97 \%$ (isotactic triad) and $r r=87 \%$ (syndiotactic triad), respectively. Molecular weights of the samples were determined by
GPC measurements, using a JASCO FLC-A10 chromatography equipped with a Shodex column A-80 $(50 \mathrm{~cm} \times 2)$. Number-average molecular weight $\bar{M}_{n}$, and the ratio of weight-average molecular weight $\bar{M}_{w}$ to $\bar{M}_{n}$, $\bar{M}_{w} / \bar{M}_{n}$, were obtained as $\bar{M}_{n}=3.30 \times 10^{4}$ and $\bar{M}_{w} / \bar{M}_{n}=1.25$ for the iso-PMMA, and $\bar{M}_{n}=$ $3.41 \times 10^{4}$ and $\bar{M}_{w} / \bar{M}_{n}=1.22$ for the synPMMA. Table I shows the characteristics of the polymer samples.

\section{Measurements of Dielectric Constants and Densities}

Dielectric constants were measured with a General Radio Co. 1620A type capacitance bridge at a frequency $10 \mathrm{kHz}$. The electrodes used for measurement is a three-terminal type, consisting of platinum electrodes of coaxial cylinders set in a double-walled pyrex cell. The capacitance of the cell with air is $38.32 \mathrm{pF}$. The details of the apparatus were reported elsewhere. ${ }^{17 a}$ During measurement, the cell was immersed in a silicone oil-bath whose temperature was controlled to $\pm 0.01^{\circ} \mathrm{C}$. Benzene was used a solvent for measurement. It was dried by passing through Molecular Sieve $4 \mathrm{~A}$ and carefully purified by repeated distallation before use. Measurements were carried out at weight fractions from 0.005 to 0.025 for each sample over the temperature range from 25 to $55^{\circ} \mathrm{C}$.

The densities of the polymer solutions were determined with a flask-type capillary pycnometer of $c a .10 \mathrm{ml}$ volume.

Table I. Characteristics of PMMA samples

\begin{tabular}{|c|c|c|c|c|c|c|}
\hline \multirow{2}{*}{ Polymer } & \multirow{2}{*}{ Initiator } & \multicolumn{3}{|c|}{ Tacticity $/ \%$} & \multirow{2}{*}{$\bar{M}_{n}^{\mathrm{c}}$} & \multirow{2}{*}{$\bar{M}_{w} / \bar{M}_{n}^{\mathrm{c}}$} \\
\hline & & $\mathrm{mm}$ & $m r$ & $r r$ & & \\
\hline iso-PMMA ${ }^{\mathrm{a}}$ & $t-\mathrm{C}_{4} \mathrm{H}_{9} \mathrm{MgBr}$ & 97 & 2 & 1 & 33,000 & 1.25 \\
\hline syn-PMMA ${ }^{b}$ & $i-\mathrm{C}_{4} \mathrm{H}_{9} \mathrm{Li}-\left(\mathrm{C}_{2} \mathrm{H}_{5}\right)_{3} \mathrm{Al}$ & 1 & 12 & 87 & 34,100 & 1.22 \\
\hline
\end{tabular}

a Prepared in toluene at $-60^{\circ} \mathrm{C}$.

b Prepared in toluene at $-78^{\circ} \mathrm{C},[\mathrm{Li} / \mathrm{Al}]=1 / 3 \mathrm{~mol} / \mathrm{mol}$.

c Determined by GPC. 


\section{RESULTS AND DISCUSSION}

The results of dielectric constant and density measurements are given in Table II. Dipole moments per constitutional repeating unit of PMMA were calculated by the conventional method on the basis of the Debye theory, ${ }^{18}$ from the experimental data of dielectric constants and densities.

The specific polarization of the polymers at infinite dilution $\left(p_{2}\right)$ was calculated using the Halverstadt-Kumler equation ${ }^{9}$ :

$$
p_{2}=p_{1}\left(1+\frac{3 \alpha}{\left(\varepsilon_{1}-1\right)\left(\varepsilon_{2}+2\right)}-\frac{\beta}{\rho_{1}}\right)
$$

with $\alpha=\mathrm{d}\left(\varepsilon_{12}-\varepsilon_{1}\right) / \mathrm{d} w$ and $\beta=\mathrm{d}\left(\rho_{12}-\rho_{1}\right) / \mathrm{d} w$, where $w$ is the weight fraction of solute (polymer), $\varepsilon$ dielectric constant, $\rho$ density, and the suffixes 1, 2, and 12 refer to solvent, solute, and solution, respectively.

The specific polarization $p_{2}$ multiplied by molecular weight $M_{0}$ of the monomeric unit gives the molecular polarization, $P_{2}\left(=M_{0} p_{2}\right)$. Thus, the average dipole moment per constitutional repeating unit $\mu$ was calculated by the equation:

$$
\begin{aligned}
\mu= & \left\{\frac{9 k T}{4 \pi N_{\mathrm{A}}}\left(P_{2}-R_{\mathrm{m}, \mathrm{D}}\right)\right\}^{1 / 2} \\
& =0.01281\left\{\left(P_{2}-R_{\mathrm{m}, \mathrm{D}}\right) T\right\}^{1 / 2}
\end{aligned}
$$

where $k$ the Boltzmann constant, $N_{\mathrm{A}}$ the Avogadro number, and $T$, temperature. In eq 2 , the sum of electronic and atomic polarizations was assumed to be equal to the molecular refraction for the D line, $R_{\mathrm{m}, \mathrm{D}}$, which was

\begin{tabular}{|c|c|c|c|c|c|c|}
\hline$\frac{\text { Temp }}{{ }^{\circ} \mathrm{C}}$ & $\alpha$ & $\frac{\beta}{\mathrm{gcm}^{-3}}$ & $\frac{p_{2}}{\mathrm{~cm}^{3} \mathrm{~g}^{-1}}$ & $\frac{P_{2}}{\mathrm{~cm}^{3} \mathrm{~mol}^{-1}}$ & $\frac{\mu^{\mathrm{a}}}{\text { Debye }}$ & $D_{\mathrm{r}}^{\mathrm{b}}$ \\
\hline 25 & 2.377 & 0.2759 & 0.6802 & 68.10 & 1.457 & 0.710 \\
\hline 30 & 2.328 & 0.2787 & 0.6742 & 67.50 & 1.459 & 0.712 \\
\hline 35 & 2.280 & 0.2815 & 0.6680 & 66.88 & 1.461 & 0.714 \\
\hline 40 & 2.231 & 0.2842 & 0.6617 & 66.25 & 1.461 & 0.714 \\
\hline 45 & 2.182 & 0.2870 & 0.6552 & 65.60 & 1.461 & 0.714 \\
\hline 50 & 2.134 & 0.2898 & 0.6486 & 64.94 & 1.461 & 0.714 \\
\hline 55 & 2.085 & 0.2925 & 0.6418 & 64.26 & 1.460 & 0.714 \\
\hline
\end{tabular}
calculated from the atomic refractions of the

Table IIa. Dipole moments of iso-PMMA measured in benzene at various temperatures

\begin{tabular}{|c|c|c|c|c|c|c|}
\hline Temp & \multirow{2}{*}{$\alpha$} & $\beta$ & $p_{2}$ & $P_{2}$ & $\mu^{\mathrm{a}}$ & \multirow{2}{*}{$D_{\mathrm{r}}^{\mathrm{b}}$} \\
\hline${ }^{\circ} \mathrm{C}$ & & $\mathrm{g} \mathrm{cm}^{-3}$ & $\mathrm{~cm}^{3} \mathrm{~g}^{-1}$ & $\mathrm{~cm}^{3} \mathrm{~mol}^{-1}$ & Debye & \\
\hline 25 & 2.009 & 0.2938 & 0.6041 & 60.49 & 1.323 & 0.568 \\
\hline 30 & 1.981 & 0.2865 & 0.6051 & 60.59 & 1.336 & 0.597 \\
\hline 35 & 1.953 & 0.2791 & 0.6061 & 60.69 & 1.349 & 0.609 \\
\hline 40 & 1.924 & 0.2718 & 0.6071 & 60.78 & 1.362 & 0.620 \\
\hline 45 & 1.896 & 0.2645 & 0.6080 & 60.88 & 1.374 & 0.631 \\
\hline 50 & 1.867 & 0.2572 & 0.6089 & 60.97 & 1.387 & 0.643 \\
\hline 55 & 1.839 & 0.2498 & 0.6098 & 61.05 & 1.399 & 0.655 \\
\hline
\end{tabular}

Table IIb. Dipole moments of syn-PMMA measured in benzene at various temperatures

a Dipole moment per repeating unit in PMMA, calculated by eq $2, \mu=0.01281\left\{\left(P_{2}-R_{\mathrm{m}, \mathrm{D}}\right) T\right\}^{1 / 2}$, in which $24.72 \mathrm{~cm}^{3}$ was used as the value of $R_{\mathrm{m}, \mathrm{D}}$.

b $D_{\mathrm{r}}=\left\langle m^{2}\right\rangle / n \mu_{0}^{2}=\left\langle\mu^{2}\right\rangle / \mu_{0}^{2}$ is the dipole moment ratio, where $\mu_{0}$ is the dipole moment of the repeating unit and $n$, the number of units. The dipole moment of methyl propionate, $1.73 \mathrm{D}$, was used as $\mu_{0}$. 


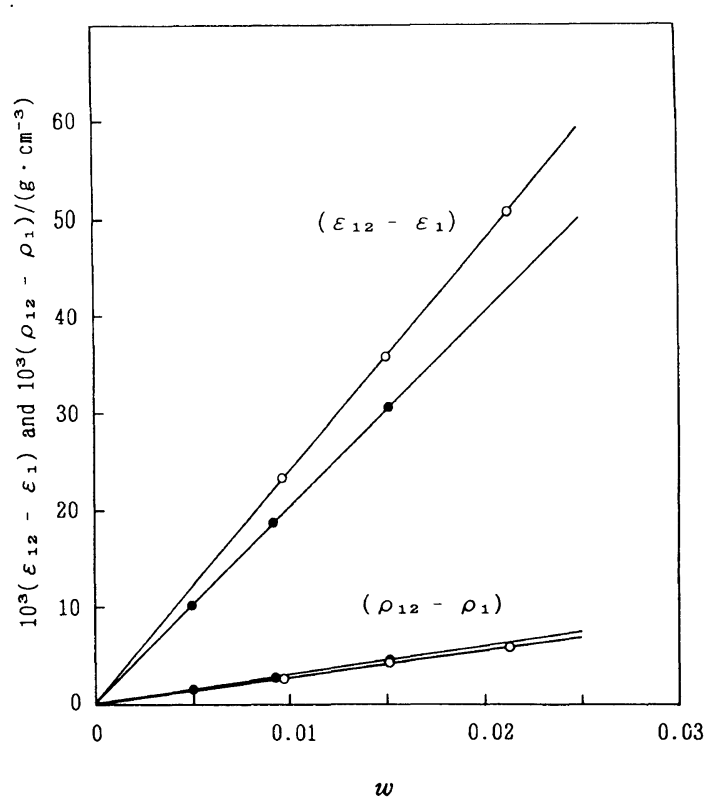

Figure 1. Plots of $\left(\varepsilon_{12}-\varepsilon_{1}\right)$ versus $w$ and Plots of $\left(\rho_{12}-\rho_{1}\right)$ versus $w$ for iso-PMMA $(\bigcirc)$ and syn-PMMA (O) in benzene at $25^{\circ} \mathrm{C}$ : $\varepsilon$, dielectric constant; $\rho$, density; $w$, weight fraction of the polymer).

constituents.

Dipole moments evaluated for the iso- and syn-PMMA samples are given in Tables IIa and IIb, together with $P_{2}, \alpha$, and $\beta$. Specific increments at infinite dilution, $\alpha$ and $\beta$, were determined from plots of $\left(\varepsilon_{12}-\varepsilon_{1}\right)$ versus $w$ and those of $\left(\rho_{12}-\rho_{1}\right)$ versus $w$, respectively. Figure 1 shows an example of the plots. It was found that the values of the dipole moments obtained for the two PMMA samples are appreciably different from each other as could be expected. The $\mu$ values of the iso-PMMA are greater than those of the syn-PMMA, e.g., the values of the dipole moment obtained in benzene at $25^{\circ} \mathrm{C}$ are $1.457 \mathrm{D}$ for the iso-PMMA and $1.323 \mathrm{D}$ for the syn-PMMA.

In Table III, the dipole moments of PMMA reported in the literatures are listed for comparison. This table shows that the values of $\mu$ obtained in this study are in general agreement with those reported previously and the dipole moment of atactic PMMA measured sometime ago by one of the present authors
Table III. Dipole moments of PMMA reported in literature

\begin{tabular}{|c|c|c|c|c|}
\hline \multirow{2}{*}{ Polymer } & \multirow{2}{*}{ Solvent } & $t$ & $\mu$ & \multirow{2}{*}{ Reference } \\
\hline & & ${ }^{\circ} \mathrm{C}$ & Debye & \\
\hline \multirow[t]{3}{*}{ atac-PMMA } & Benzene & 10 & $1.36\}$ & \multirow{3}{*}{$\begin{array}{l}\text { M. Shima } \\
\text { (1962) } \\
\text { J. Marchal } \\
\text { et al. }{ }^{3}(1958)\end{array}$} \\
\hline & Benzene & 40 & $1.41\}$ & \\
\hline & Benzene & 25 & 1.38 & \\
\hline iso-PMMA & Benzene & 30 & 1.43 & \multirow{2}{*}{$\begin{array}{l}\text { H. A. Pohl } \\
\text { et al. }^{4}(1960)\end{array}$} \\
\hline syn-PMMA & Benzene & 30 & $1.27\}$ & \\
\hline iso-PMMA & Benzene & 25 & $1.51\}$ & \multirow{2}{*}{$\begin{array}{l}\text { R. Salovey }{ }^{5} \\
\text { (1961) }\end{array}$} \\
\hline syn-PMMA & Benzene & 25 & $1.45\}$ & \\
\hline \multirow[t]{2}{*}{ iso-PMMA } & Toluene & 25 & 1.34 & \multirow{4}{*}{$\begin{array}{l}\text { A. Roig et al. } \\
\quad(1968)\end{array}$} \\
\hline & Toluene & 40 & 1.35 & \\
\hline \multirow[t]{2}{*}{ syn-PMMA } & Toluene & 25 & 1.30 & \\
\hline & Toluene & 40 & 1.32 & \\
\hline iso-PMMA & Benzene & 25 & 1.457 & \multirow{4}{*}{ Present study } \\
\hline & Benzene & 40 & 1.461 & \\
\hline \multirow[t]{2}{*}{ syn-PMMA } & Benzene & 25 & 1.323 & \\
\hline & Benzene & 40 & 1.362 & \\
\hline
\end{tabular}

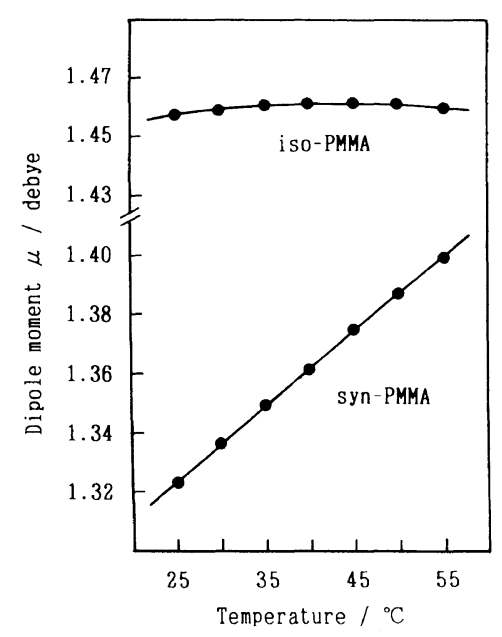

Figure 2. Temperature dependence of dipole moments $\mu$ for iso-PMMA and syn-PMMA in benzene.

(M.S.) is midway between the values for iso- and syn-PMMAs.

Figure 2 shows the temperature dependence of the dipole moments of iso- and syn-PMMAs. It is noted that temperature dependence is markedly different for iso-PMMA and synPMMA. By raising the temperature from 25 to $55^{\circ} \mathrm{C}$, the $\mu$ value of the iso-PMMA showed 
little change, and remained nearly constant, whereas that for syn-PMMA increased continuously; e.g., $1.457 \mathrm{D}$ (at $25^{\circ} \mathrm{C}$ ) and $1.460 \mathrm{D}$ (at $55^{\circ} \mathrm{C}$ ) were obtained for the iso-PMMA and, $1.323 \mathrm{D}\left(\right.$ at $25^{\circ} \mathrm{C}$ ) and $1.399 \mathrm{D}$ (at $55^{\circ} \mathrm{C}$ ) for the syn-PMMA, giving the temperature coefficient, $\mathrm{d} \ln \left\langle\mu^{2}\right\rangle / \mathrm{d} T=3.72 \times 10^{-3} \mathrm{~K}^{-1}$. The difference in dipole moments of the iso- and syn-PMMA should be caused by orientational differences of the polar side groups in the respective PMMA chain. It is known that there is much difference in the properties of iso- and syn-PMMAs in solid. The glass transition temperature of syn-PMMA is much higher than that of iso-PMMA, ${ }^{12}$ and iso-PMMA is more soluble in benzene than syn-PMMA. From these observations, it is probable that segmental motions including internal rotation of the polar side groups in PMMA chains are more restricted in syn-PMMA than in iso-PMMA. A marked temperature dependence of the dipole moment of syn-PMMA shown in Figure 2 might be explained by considering the conditions of restricted rotation of polar side groups in the molecular chain. Figure 3 shows dipole moment ratios $D_{\mathrm{r}}$ for the iso- and syn-PMMAs as a function of

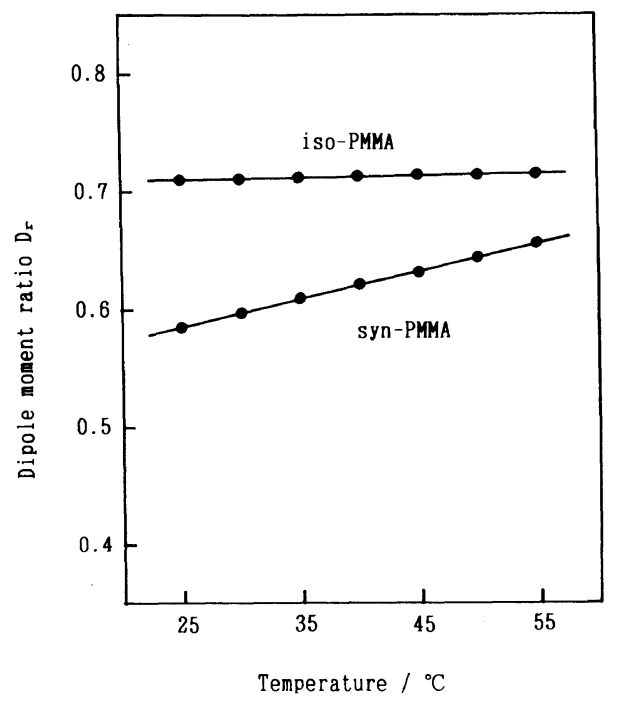

Figure 3. Dipole moment ratios $D_{\mathrm{r}}$ as a function of temperature, for iso-PMMA and syn-PMMA in benzene. temperature. The dipole moment ratio, $D_{\mathrm{r}}=$ $\left\langle m^{2}\right\rangle / n \mu_{0}^{2}=\left\langle\mu^{2}\right\rangle / \mu_{0}^{2}$, is a parameter often used in conformational analysis of polymer chains and an analogue of the characteristic ratio, $C_{\mathrm{r}}=\left\langle r_{0}^{2}\right\rangle / n l^{2}$, of chain dimensions, where $\left\langle m^{2}\right\rangle$ is the mean-square dipole moment of the polymer chain with $n$ bond dipoles in magnitude $\mu_{0}$ in the chain, and $\left\langle r_{0}^{2}\right\rangle$, the mean-square end-to-end distance of the unperturbed chain with $n$ bonds of length $l$.

\section{Comparison of Experimental and Calculated Results}

A number of theoretical studies of conformational statistics of stereoregular PMMA chains has been reported. ${ }^{8-10}$ Birshtein et al. ${ }^{11}$ calculated the mean-square dipole moment $\left\langle m^{2}\right\rangle$ of iso- and syn-PMMAs by using Flory's $\operatorname{method}^{7}$ on the basis of the "rotational isomeric state" approximation. Figure 4 illustrates a part of the iso-PMMA chain, in which $\phi$ is the rotational angle around $\mathrm{C}-\mathrm{C}$ bond of the chain skeleton and $\chi$, the angle with rotation of the polar side group (methyl ester). In the calculation, they used geometrical and conformation energetic parameters, previously obtained by Flory and coworker. ${ }^{8}$ The bond angles they used are indicated in Figure 4. With respect to rotation around the $\mathrm{C}-\mathrm{C}$ bond of the chain skeleton, they chose two rotational isomeric states, trans $(t)$ and gauche $(g)$, as preferential, excluding " $g$ '" because it has a high conformation energy. Then, the internal rotation characteristics were described by matrices of statistical weight of the order

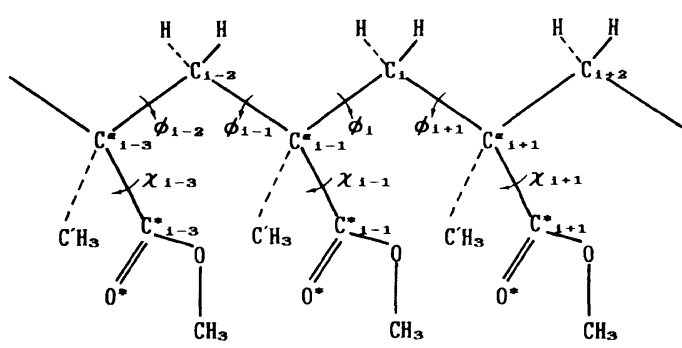

Figure 4. A part of iso-PMMA chain. $\angle C^{\alpha} \mathrm{C}=110^{\circ}$; $\angle \mathrm{C}^{\alpha} \mathrm{CC}^{\alpha}=122^{\circ} ; \angle \mathrm{C}^{\prime} \mathrm{C}^{\alpha} \mathrm{C}^{*}=109.5^{\circ}$. 
$2 \times 2$; the $U^{\prime}$ matrix for rotations in the vicinity of a pair of bonds adjoining $\mathrm{C}^{\alpha}$,

$$
\boldsymbol{U}^{\prime}={ }_{g}^{t} \mid \frac{t}{\left(\begin{array}{ll}
1 & 1 \\
1 & 0
\end{array}\right)}
$$

and the matrix $U^{\prime \prime}$ for rotation of bonds between neighboring $\mathrm{C}^{\alpha}$,

$$
\begin{gathered}
\boldsymbol{U}_{\text {iso }}^{\prime \prime}={ }_{g} \mid \frac{t}{\left(\begin{array}{cc}
1 & a \\
a & a^{2} / b
\end{array}\right)} \\
\boldsymbol{U}_{\mathrm{syn}}^{\prime \prime}={ }_{g}^{t} \mid \begin{array}{cc}
t & g \\
\left(\begin{array}{cc}
b & a \\
a & a^{2} / b
\end{array}\right)
\end{array}
\end{gathered}
$$

The values of the parameters previously determined by Flory and coworkers are

and

$$
a=1.6 \exp \left(-E_{\mathrm{a}} / R T\right)
$$

$$
b=1.4 \exp \left(-E_{\mathrm{b}} / R T\right)
$$

where $E_{\mathrm{a}}=1.1 \mathrm{kcal} \mathrm{mol}^{-1}$, and $E_{\mathrm{b}}=-0.6 \mathrm{kcal}$ $\mathrm{mol}^{-1}$ at $300 \mathrm{~K}$. Then, $a=0.26$ and $b=3.8$.

Using these energetic parameters, Birshtein et al. calculated the probability of local conformations in the PMMA chains. The results obtained showed that in the iso-PMMA chains, $t t$ conformation is predominant, giving $w_{t t}=$ $75 \%$ and the remaining $25 \%$ of the units assume a $t g(g t)$ conformation, i.e., $w_{t g(=g t)}=$ $25 \%$, and in the syn-PMMA chain, $w_{t t}=90 \%$ and $w_{t g(=g t)}=10 \% . g g$ conformations were not found in either of the iso- and syn-PMMA chains.

Rotational angle $\chi$ around $\mathrm{C}^{\alpha}-\mathrm{C}^{*}$ bond was defined as $\chi=0^{\circ}$ when $\mathrm{C}^{*}=\mathrm{O}^{*}$ bond of methyl ester groups is in a cis position toward the $\mathrm{C}^{\alpha}-\mathrm{C}^{\prime} \mathrm{H}_{3}$ bond, and $\chi=180^{\circ}$ when $\mathrm{C}^{*}=\mathrm{O}^{*}$ bond is in a trans position against the $\mathrm{C}^{\alpha}-\mathrm{C}^{\prime} \mathrm{H}_{3}$ bond. The dipole moment $\left(\mu_{0}=1.80 \mathrm{D}\right)$ of a methyl ester group in the constitutional unit of PMMA chain was assumed to be directed along the $\mathrm{C}^{*}=\mathrm{O}^{*}$ bond. Under these conditions, the dipole moment ratio, $D_{\mathrm{r}}=\left\langle\mu^{2}\right\rangle / \mu_{0}^{2}$, was calculated for three cases. Assumptions made for the respective case are as follows;

Case (1): free rotation of methyl ester group around $\mathrm{C}^{\alpha}-\mathrm{C}^{*}$ bond is assumed.

Case (2): rotation of methyl ester group is restricted, and fixed at $\chi=0$ or $180^{\circ}$.

Case (3): rotational angle of methyl ester group alternates $\chi=0^{\circ}$ and $\chi=180^{\circ}$, and the probability of alternation, $w_{x}$, is introduced.

In case (1), the results of calculation gave large values of $D_{\mathrm{r}}(>1)$ for both of iso- and syn-PMMAs. Experimental values of $D_{\mathrm{r}}$ obtained for iso- and syn-PMMA chains are always less than $1\left(D_{\mathrm{r}}=0.57-0.71\right)$ as shown in Table II, and also in previous papers. ${ }^{4-6}$ The assumption in case (1) is thus to be rejected.

Under the assumptions in cases (2) and (3), the results of the calculation lead to the conclusion that $D_{\mathrm{r}}$ for syn-PMMA becomes less than 1 , only if $\chi$ is fixed at $180^{\circ}$ and $w_{x}=0$, or it alternates $\chi=0^{\circ}$ and $\chi=180^{\circ}$ with the probability, $w_{x},\left(1 / 2 \lesssim w_{x} \lesssim 3 / 4\right)$, while $D_{\mathrm{r}}<1$ can be obtained for iso-PMMA, only if alternation of $\chi=0^{\circ}$ and $\chi=180^{\circ}$ takes place with the probability, $w_{x},\left(w_{x}>1 / 2\right)$. In any case, the value of $D_{\mathrm{r}}$ obtained for syn-PMMA appears smaller than that for iso-PMMA.

The experimental values of $D_{\mathrm{r}}$ given in Figure 3 show similar trend of the calculated ones. $D_{\mathrm{r}}$ of the syn-PMMA was smaller than that of the iso-PMMA under the conditions of present experiments. Difference in temperature dependence of $D_{\mathrm{r}}$ between the iso-PMMA and the syn-PMMA may be interpreted according to calculation results. If the probability of the alternation $w_{x}$ for the iso-PMMA is rather large at a given temperature, it will not change much with increasing temperature, which explains the slight change of $D_{\mathrm{r}}$ for the iso-PMMA between 25 and $55^{\circ} \mathrm{C}$. On the other hand, there are two possibilities for the orientation of polar side groups in the syn-PMMA chain. The value of $D_{\mathrm{r}}(=0.6)$ obtained with the fixed rotational angle $\chi=180^{\circ}$, is smaller than that $(\simeq 0.7-0.8)$ obtained introducing the probability of alternation of the rotational angle, $\chi=0^{\circ}$ and $\chi=180^{\circ}$. The raise in temperature may allow change from the fixed value of $\chi=180^{\circ}$ to the alternation of $\chi=0^{\circ}$ and $\chi=180^{\circ}$. If this takes 
place, an increase of $D_{\mathrm{r}}$ for the syn-PMMA with increasing temperature is explainable.

The PMMA samples used in this study are highly iso-tactic and syndiotactic, respectively. However, there are some breaks in stereoregular sequences in the respective PMMA chain, which may affect the conformation of the chain as a total, and then, also the values of $\left\langle m^{2}\right\rangle$. Moreover, the dipole moments experimentally evaluated in solution might be influenced by the solvent used for measurement. Thus, it should be pointed out that the conclusions from this study is still not conclusive, and further study should be conducted.

\section{REFERENCES}

1. M. Shima, J. Polym. Sci., 56, 213 (1962).

2. A. Kotera, K. Shimomura, and M. Shima, J. Polym. Sci., C, 30, 233 (1970).

3. J. Marchal and C. Lapp, J. Polym. Sci., 27, 571 (1958).

4. (a) H. A. Pohl, R. Bacskai, and W. P. Purcell, J. Phys. Chem., 64, 1701 (1960).

(b) R. Bacskai and H. A. Pohl, J. Polym. Sci., 17, 151 (1960).

5. R. Salovey, J. Polym. Sci., 50, S7 (1961).

6. A. Roig and A. Horta, J. Polym. Sci., C, 163501 (1968).
7. P. J. Flory, "Statistical Mechanics of Chain Molecules," Interscience, New York, N.Y., 1969.

8. P. R. Sundararajan and P. J. Flory, J. Am. Chem. Soc., 96, 5025 (1974).

9. M. Vacatello and P. J. Flory, Macromolecules, 19, 405 (1986).

10. P. R. Sundararajan, Macromolecules, 19, 415 (1986).

11. T. M. Birshtein, A. A. Merkur'yeva, and A. N. Goryunov, Polym. Sci. U.S.S.R., 25, 143 (1983).

12. K. Hatada, T. Kitayama, and K. Ute, Prog. Polym. Sci., 13, 189 (1988).

13. K. Hatada, K. Ute, K. Tanaka, T. Kitayama, and Y. Okamoto, Polym. J., 17, 977 (1985).

14. K. Hatada, K. Ute, K. Tanaka, Y. Okamoto, and T. Kitayama, Polym. J., 18, 1037 (1986).

15. T. Kitayama, T. Shinozaki, E. Masuda, M. Yamamoto, and K. Hatada, Polym. Bull., 20, 505 (1988).

16. T. Kitayama, T. Shinozaki, T. Sakamoto, M. Yamamoto, and K. Hatada, Makromol. Chem., 15 (Suppl.), 167 (1989).

17. (a) N. Yamaguchi, M. Sato, and M. Shima, Polym. J., 20, 97 (1988).

(b) M. Sato, N. Yamaguchi, and M. Shima, Polym. J., 23, 735 (1991).

(c) M. Shima, M. Sato, and N. Yamaguchi, Makromol. Chem., 192, 531 (1991).

18. (a) P. Debye, "Polar Molecules," Chemical Catalog, New York, N.Y., 1929.

(b) P. Debye and F. Bueche, J. Chem. Phys., 19, 589 (1951).

19. I. F. Halverstadt and W. D. Kumler, J. Am. Chem. Soc., 64, 2988 (1942). 